\section{Easier localization of the Crosby capsule in the alimentary tract}

\author{
ALAN G. COX From the University Department \\ of Surgery, Royal Infirmary, Sheffield
}

The Crosby capsule has gained wide acceptance as a useful instrument for peroral biopsy of small intestinal mucosa but there can be uncertainty regarding its position in the alimentary tract when the radiolucent polyethylene tubing supplied in the standard kit is used. This minor difficulty can be solved by using radio-opaque polyvinyl chloride tubing ${ }^{1}$ (external diameter $=2 \mathrm{~mm}$., internal diameter $=1.4 \mathrm{~mm}$.). This is seen clearly in radiographs of the abdomen, and the characteristic curve of the tube lying in the duodenal loop leaves no doubt when the capsule has passed the pylorus (Fig. 1). An added advantage is that the tube can be easily marked at measured intervals using a ball-point pen.

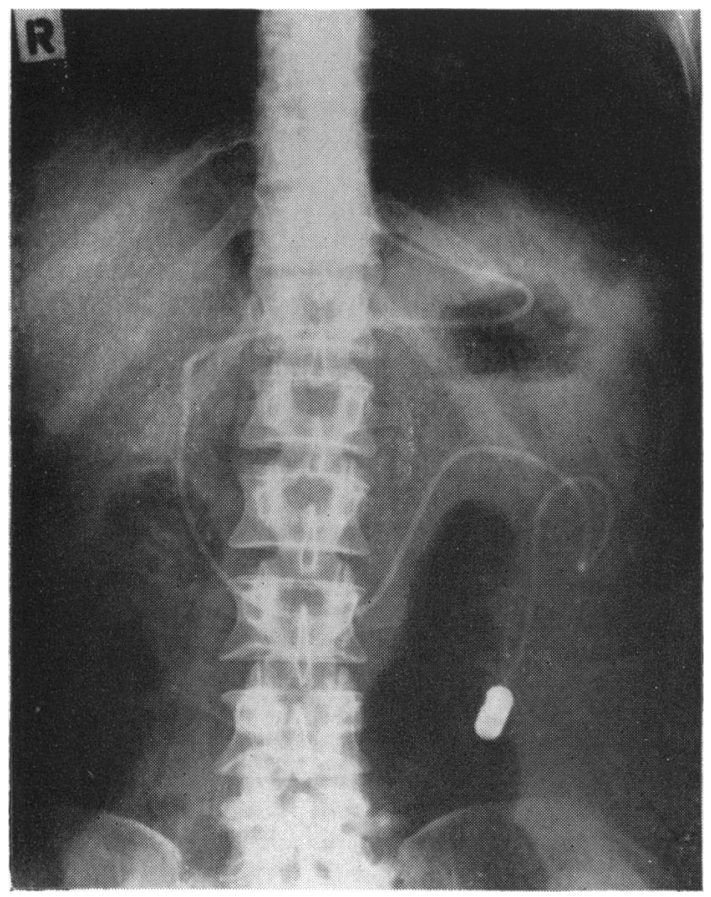

FIG. 1. Radiograph of abdomen showing the ease with which the tubing can be seen.

'Supplied by Portland Plastics Ltd.

\section{Gastroenterological Society of Australia}

A scientific session and clinical meeting was held at Prince Henry's Hospital, Melbourne, on 11 August 1962 under the Presidency of Dr. W. E. King.

The following are summaries of the papers presented:

DR. W. B. HENNESSY discussed 'Vitamin $B_{12}$ and folic acid deficiency following gastric resection' based upon his observations of nine patients (two, total gastrectomy and seven, Polya gastrectomy).

Macrocytic anaemia with megaloblastic transformation of the bone marrow occurred in two patients, five and six years after total gastrectomy, and in two patients, four and five years after partial gastrectomy. These four patients responded satisfactorily to treatment with parenteral vitamin $\mathbf{B}_{\mathbf{1 2}}$. Three patients were found to have macrocytic anaemia and partial megaloblastic transformation of the bone marrow, three, four, and eight years after operation. Two of these had low serum vitamin $B_{12}$ levels and the anaemia was cured following intramuscular vitamin $B_{12}$. The gastric mucosa in one was histologically normal. The third patient in this group had a normal serum vitamin $B_{12}$ level but the serum folic acid level was low. The haemoglobin level did not alter following injections of vitamin $B_{12}$ and oral folic acid, but returned to normal after parenteral folic acid. The remaining two patients presented with evidence of vitamin $B_{12}$ deficiency without anaemia two and four years after partial gastrectomy. The former had macrocytosis and a low serum vitamin $B_{12}$ level and the latter had advanced subacute combined degeneration of the cord.

All patients subjected to total gastrectomy should be treated subsequently with intramuscular vitamin $\mathbf{B}_{12}$; secondly, megaloblastic anaemia was much more likely to occur after a Polya gastrectomy than after a Billroth I procedure; thirdly, although folic acid deficiency might occasionally occur, loss of intrinsic factor or failure to secrete intrinsic factor was the commonest cause of megaloblastic anaemia after partial gastrectomy; and, fourthly, vitamin $\mathbf{B}_{12}$ deficiency after partial gastrectomy could occur in the presence of a histologically normal gastric mucosa, suggesting that other mechanisms might be important contributory causes of vitamin $B_{12}$ deficiency in some patients

'The incidence of peptic ulcer in Tasmania' was discussed by DR. T. KIRKLAND. In Tasmania an attempt was made to record every individual in whom an ulcer was diagnosed at the first barium meal examination during 1960-61. All types of patient were included and no distinction was made between those in whom deformity was recognized and those in whom a crater was demonstrated. Information was obtained about 165 males and 91 females from a total population of 350,340 . The largest number of men were in the fourth decade; women were most numerous in the fifth. When the figures were related to the population at risk these peaks moved to the seventh 